\title{
Experiencing Simulated Confrontations in Virtual Reality
}

\author{
Patrick Dickinson \\ School of Computer Science \\ University of Lincoln, UK \\ pdickinson@lincoln.ac.uk
}

Andrew Westerside

School of Fine \& Performing Arts

University of Lincoln, UK

awesterside@lincoln.ac.uk

Kieran Hicks

School of Computer Science

University of Lincoln, UK

khicks@lincoln.ac.uk

\author{
Arthur Jones \\ School of Computer Science \\ University of Lincoln, UK \\ artjones@lincoln.ac.uk
}

Franky Mulloy

School of Sports Science

University of Lincoln, UK

fmulloy@lincoln.ac.uk

\section{Liam Wilson}

School of Computer Science

University of Lincoln, UK

liamwilson@lincoln.ac.uk
Wayne Christian

School of Design

University of Lincoln, UK

wchristian@lincoln.ac.uk

\author{
Kathrin Gerling \\ Department of Computer Science \\ KU Leuven, Belgium \\ kathrin.gerling@kuleuven.be
}

Adrian Parke

School of Education \& Social Sciences

University of West Scotland, UK

adrian.parke@uws.ac.uk
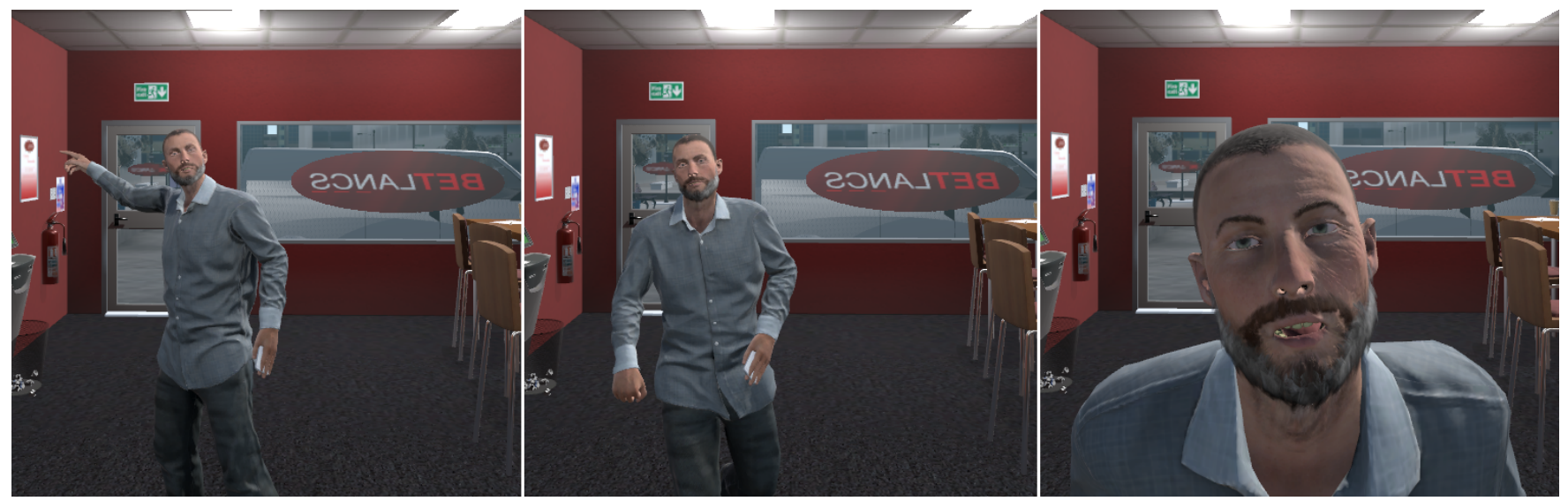

Figure 1: We compare users' responses to a confrontational virtual character in VR and on a 2D display

\begin{abstract}
The use of virtual reality (VR) to simulate confrontational human behaviour has significant potential for use in training, where the recreation of uncomfortable feelings may help users to prepare for challenging real-life situations. In this paper we present a user study $(\mathrm{n}=68)$ in which participants experienced simulated confrontational behaviour performed by a virtual character either in immersive VR, or on a 2D display. Participants reported a higher elevation in anxiety in VR, which correlated positively with a perceived sense of physical space. Character believability was influenced negatively by visual elements of the simulation, and positively by behavioural
\end{abstract}

Permission to make digital or hard copies of all or part of this work for personal or classroom use is granted without fee provided that copies are not made or distributed for profit or commercial advantage and that copies bear this notice and the full citation on the first page. Copyrights for components of this work owned by others than ACM must be honored. Abstracting with credit is permitted. To copy otherwise, or republish, to post on servers or to redistribute to lists, requires prior specific permission and/or a fee. Request permissions from permissions@acm.org.

CHI '21, May 8-13, 2021, Yokohama, Japan

(c) 2021 Association for Computing Machinery.

ACM ISBN 978-1-4503-8096-6/21/05 . .\$15.00

https://doi.org/10.1145/3411764.3445401 elements, which complements findings from previous work. We recommend the use of VR for simulations of confrontational behaviour, where a realistic emotional response is part of the intended experience. We also discuss incorporation of domain knowledge of human behaviours, and carefully crafted motion-captured sequences, to increase users' sense of believability.

\section{CCS CONCEPTS}

- Human-centered computing $\rightarrow$ Virtual reality; User studies.

\section{KEYWORDS}

Virtual reality, confrontational behaviour, virtual character

\section{ACM Reference Format:}

Patrick Dickinson, Arthur Jones, Wayne Christian, Andrew Westerside, Franky Mulloy, Kathrin Gerling, Kieran Hicks, Liam Wilson, and Adrian Parke. 2021. Experiencing Simulated Confrontations in Virtual Reality. In CHI Conference on Human Factors in Computing Systems (CHI '21), May 8-13, 2021, Yokohama, Japan. ACM, New York, NY, USA, 10 pages. https: //doi.org/10.1145/3411764.3445401 


\section{INTRODUCTION}

Virtual characters are widely used in virtual reality (VR) applications, and previous work has shown that, in some respects, users respond to them in similar ways as they do to real people [13, 20, $31,50]$. This affords the potential for VR to be used for training, or other applications, that simulate believable real-life interactions between people.

The use of VR simulations to train people how to deal with challenging or confrontational behaviours has been previously described; for example, helping doctors to deal with disagreeable patients [33]. In real-life, such situations can create uncomfortable feelings, and replicating this discomfort enables trainees to prepare for such situations [3]. However, to date, relatively little work has examined the emotional responses of users experiencing confrontational behaviour by virtual characters in VR simulations, and none has considered whether VR simulations are more able to recreate such responses than those presented on 2D displays.

Questions around the believability of virtual human characters in VR have focussed on the challenge of perceived intelligence (along the lines of the Turing Test) $[3,32]$, uncanniness of realistic-looking characters [51], and use of other sensations $[4,18]$. However, attention has been drawn to other factors which may affect the believability of simulations of emotive, confrontational, or challenging situations, such as perceived threat, and credibility of behaviour and dialogue [4, 44]. Methods for the construction of believable, convincing simulations of confrontational behaviour have not yet been explored, and warrant further study to help designers create effective experiences.

In this paper we address both of these issues. We present the results of our study, in which we firstly compare participants' experiences of a virtual character engaging in confrontational behaviour in a VR simulation, with those experiencing the same behaviour represented on a large $2 \mathrm{D}$ screen. We focus on resulting feelings of anxiety and presence, quantified using self-report measures. We further explore the believability of confrontational characters. To do this we designed our study conditions around motion captured performances based on real-life documented behaviours. To this end, we have contextualised our study in a specific hypothetical training scenario, set in a UK gambling shop, and formed a multi-disciplinary research team which includes expertise in the behaviours we have modelled, and experience in live performance-based research. We elicit participants' perceptions of believability in both VR and 2D using semi-structured interviews and qualitative analysis.

Our findings show that simulations of confrontational behaviour are able to evoke feelings of anxiety when experienced on both 2D screens and VR, but are more highly elevated in VR, and also correlate positively with participants' sense of physical space. We also show that participants' perceptions of character believability is influenced by a range of factors: behavioural features may contribute positively, even in unfamiliar contexts, whereas visual elements were often seen to undermine believability. We conclude by discussing implications and trade-offs for the design of such VR experiences, and we also discuss ethical considerations when using VR to simulate experiences that may evoke feelings of anxiety or stress.

\section{EXISTING WORK}

The concept of presence describes the sensation of being physically located within a simulated environment. It is most closely associated with immersive VR, but may also be experienced in other interactive systems, such as traditional 2D displays (e.g. [25, 40]), or high-definition television [6]. Presence is a defining feature of VR experiences, and much work has sought to deconstruct and understand its effects on users' experiences. Slater [42] introduced the terms place illusion, which refers to the sensation of "being there", and plausibility illusion, which refers to the sense that what is happening in the simulation is real, and suggested that plausibility illusion is dependent on a number of factors including "the notion of the credibility of events in comparison with what would be expected in reality in similar circumstances". In our work we explore participants' perceptions of the believability of a confrontational character: themes arising from our analysis overlap with the concept of plausibility illusion, which we take as a reference point. Much previous work has explored factors which affect presence, including immersive platform characteristics [11], visual fidelity [26], and interface design [46]: it is also related to users' emotional responses in VR, which is a central interest of our work.

\subsection{Emotional Responses of Users in Virtual Reality}

The capacity for VR to elicit emotional responses has been established for some time. For example, it has been shown that virtual reality exposure therapy (VRET) can be as effective as in vivo therapy $[9,47]$. While the therapeutic effectiveness of VRET is wellestablished, less work has examined users' emotional response in other types of VR applications. Some studies have reported a correlation between sense of presence and emotional response [38], most commonly in clinical/therapeutic applications (e.g. [1, 5]). However, results are more varied in non-therapeutic settings [15, 37], suggesting a more complex inter-relationship.

A small number of studies have compared emotional response in VR with the same stimulus presented on a 2D screen. For example, Estupiñán et al. [16] found that users viewing images from the Geneva Affective Picture Database [12] in a HMD self-reported higher levels of arousal than had been previously recorded in 2D. Kim et al [19] also found that users performing a Stroop task consistently experienced higher levels of arousal in VR, under varying stress levels. However, results linking levels of immersion and emotion in VR are not universally reported (e.g. [22]), again hinting at subtle inter-relationships, not yet explored in the context of virtual characters. Together, this motivates the platform comparison (VR vs 2D display) presented in our own work.

\subsection{Simulated Characters and Confrontational Behaviour}

A number of researchers have investigated users' emotional and behavioural responses to simulated human characters in VR. For example, responses to close proximity have been shown to be comparable to those seen in real-life [17], resulting in feelings of discomfort when personal space is encroached upon [8, 14, 23, 50]. Users may adapt their behaviour accordingly [29], and report preferences for additional interactions, such as eye contact [28]. 
Of particular interest, Rovira et al. [39] reported on the pilot of system used to investigate pro-social responses to witnessed aggression between simulated characters, using a CAVE system. They used motion capture to produce the character animations, but participant feedback from their study shows that some found the experience lacked credibility. The same authors presented the completed study in Slater et al. [44], and reported that users were more likely to attempt to intervene if they felt an affinity for the victim; however, some participants again raised questions about the credibility of the experience, mentioning a number of issues such as setting, dialogue, and interactivity. The authors reflected, in their discussion, on the need for more authentic reproduction of the scenario, which motivates our own approach to designing and creating our study conditions.

A small number of examples of the use of virtual environments (using either VR or 2D displays) to support training for dealing with difficult or confrontational situations have been described, such as systems for law enforcement training [10,27], public transport employees [3], and doctors [33]. A key idea is that this type of training evokes an emotional response comparable to a real-life situation. For example, Bosse et al. [3] provided feedback to users on their emotional state, using physiological measures, and evidenced that responses to confrontational simulations on 2D displays were similar to, though less intense than, real-life equivalents (these results are discussed in more detail in [2]). Bosse et al. [4] found that participants in VR experienced higher physiological stress when they believed that a virtual character could deliver an electric shock, and reported associations between believability and anxiety.

In summary, little work has yet examined users' experiences of simulations of confrontational behaviour in VR. Work using 2D displays has suggested that responses are comparable, but less intense than real-life, whilst others have raised the question of how to make such simulations in VR seem believable to users. In our work we investigate the ability of VR to provoke stronger emotional responses than 2D displays, and explore issues of perceived believability through study conditions designed to represent authentic contextualised behaviour.

\section{STUDY OVERVIEW}

Our motivation is to investigate the emotional response of users experiencing simulated confrontational behaviour in VR, particularly from the perspective of applications to training for real-life situations. Study conditions which compare simulations with performed real-life behaviours are difficult to construct and undertake with significant numbers of participants, and may not be robust due to variations in individual experience. Our study therefore compares the responses of participants to simulated confrontational behaviour in VR with the same simulation displayed on a 2D screen, noting that previous work suggests that simulations on 2D screens elicits a less intense response than live performance [2]. We address the following research questions:

- RQ1: Do users experience a more elevated emotional response to confrontational behaviour in VR, as compared to a $2 \mathrm{D}$ display?
- RQ2: Do users experience an increased sense of presence, when engaged in such experiences in VR, as compared to a $2 \mathrm{D}$ display?

- RQ3: What elements of their experience affects participants' perceptions of believability, and can carefully designed behaviours contribute positively to believability?

To address these we designed a two-condition, between subjects study, where participants in one group experienced a sequence of confrontational behaviour performed by a virtual character in a VR simulation, and the other experienced the same sequence displayed on a large 2D screen. We used a between subjects design as we felt that participants' emotional responses might be significantly altered by repeated exposure. In the remainder of this section we describe the process that we used to produce the simulated behaviour and study conditions, and conclude by introducing our measures and hypotheses.

\subsection{Context}

The simulation that we have designed recreates confrontational behaviour by a customer in a reproduction of a UK betting shop, and we suggest that such a simulation could form part of a hypothetical VR training system for employees. We have chosen this context for a number of reasons. Challenging behaviours are a commonly encountered hazard for staff working in such shops. Many of the 7000 betting shops in the UK are operated by single staff members who deal directly with the consequences of problem gambling [30]. Additionally, the characteristics of such behaviours by gamblers has been studied and documented [34-36], providing us with a framework describing a range of authentic behaviours, from which to draw an appropriate study condition.

We constructed a scenario in which the participant undertakes the role of an employee in a fictitious shop. We used a typical shop layout, as shown in Figure 2. The environment has a number of slot machine terminals, which is usual in UK betting shops. There are also video screens on one wall: during our study conditions, ambient video and audio, representative of those shown in real betting shops, were played, in order to create a sense of atmosphere. The confrontational behaviour was performed by a virtual character representing a frustrated gambler (there were no other characters present), and lasts about three minutes. During both conditions, participants were asked to stand in a specific position, and to observe the behaviour of the character (either in VR or on a 2D screen mounted at approximately head height). The character's behaviour was motion captured, so it cannot respond to actions performed by the participant; however, the character does look at the participant during the earlier parts of the sequence, and later on walks towards them, and speaks to them directly. Noting that a sense of embodiment may be maintained despite visual disimilarities from the real self $[24,45,48]$, we included a partial self-avatar in the VR condition which we dressed in a way consistent with a betting shop employee, and which we designed to appear of indeterminant gender, with a mid-range skin tone.

\subsection{Production of the Animated behaviour}

We aimed to create character behaviour based on the real observed behaviour of gamblers, using an iterative process of motion capture 


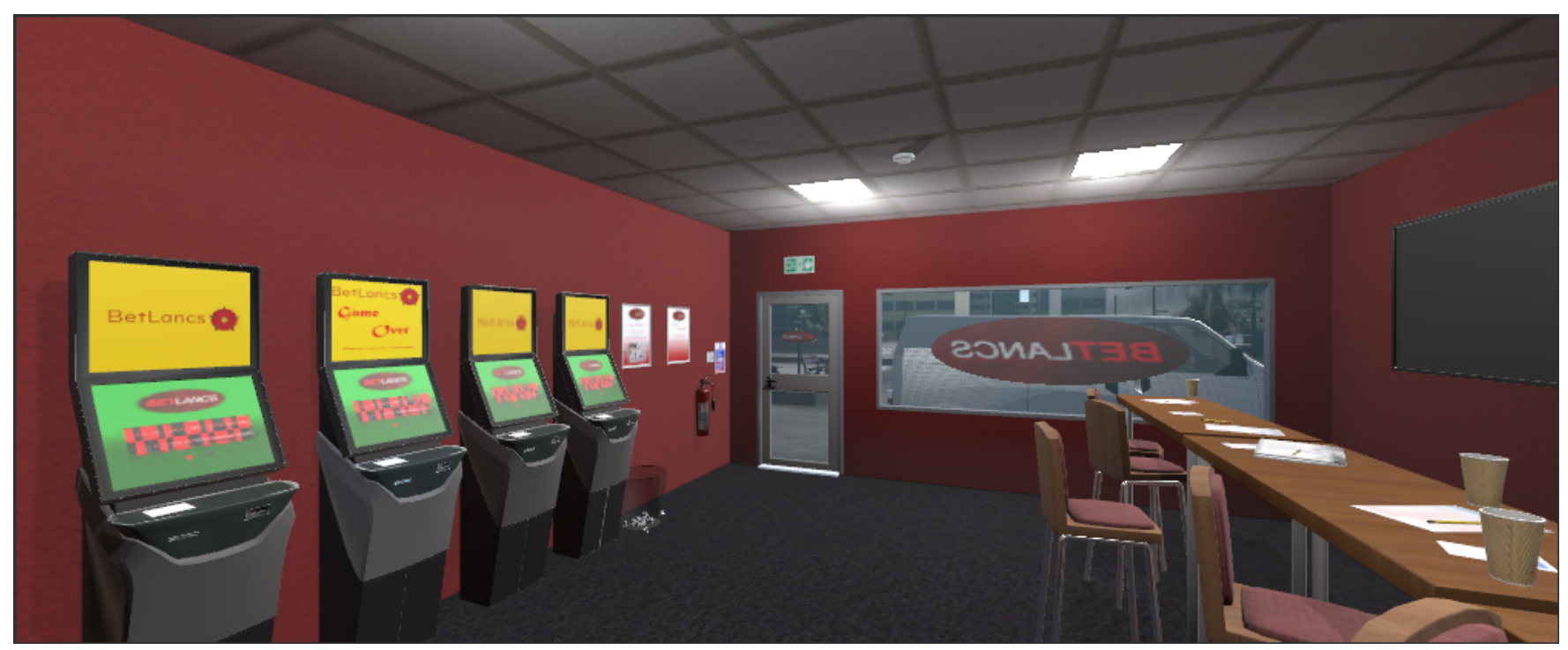

Figure 2: The betting shop virtual environment

(mocap) recording, and review. The research team included expertise in live performance acting, and also in the behaviours of slot machine gamblers. We used a Motion Analysis Cortex ${ }^{T M}$ motion capture system, comprising twelve Raptor cameras which were distributed in a circular fashion around a central point. The system was used to record three sessions, each of duration 1-2 hours. Prior to the first session, we developed an initial script and scene directions, based on observed real world behaviours [35, 36], in a performance structure which aligned with our study objectives.

Over the three sessions we recorded multiple takes of the scripted behaviour, collectively reviewing and refining the performance between sessions. We used props as a guide, and recorded video and live audio as well as motion capture data. We finally combined two segments from two different takes to create the final sequence, based on quality and duration of performance.

The final sequence was post-processed in Unity3D (Version 2018.3.4f1) to remove capture errors and noise. It was then applied to a human character model which was constructed using Adobe Fuse. The model was designed to represent a demographic commonly seen in UK betting shops (male, white, middle aged) [49], with a height of $180 \mathrm{~cm}$. The final motion sequence contained some minor noise artefacts which we could not completely remove during post-processing. Facial animations were applied using LipSynch Pro (v1.45) to generate approximate mouth animations from the recorded audio. We also added a run-time script to control the gaze direction of the model's eyes, to enhance the perception that the character was looking at the participant at various points in the sequence.

The final behaviour comprised two minutes of build up, during which the character played a gambling machine, and displayed signs of frustration (looking up, stepping back from the machine, dismissive gestures). The character also glanced at the participant on occasion during this phase, to help build a sense of co-presence $[28,41]$. The final minute (second phase) begins when the character shouts at the machine, and hits it with his fist. He then walks towards the participant in an agitated state, and demands the return of the money he has lost. The character uses a loud voice, swear words, and threatens to damage the machine, but does not threaten the participant, although the demeanour is confrontational. Images representing the confrontational phase of sequence are shown in Figure 1.

\subsection{Balancing Perceived Proximity Between Conditions}

Previous work has shown that close proximity in VR may induce feelings of discomfort (e.g. [14, 23, 50]). We therefore configured our two conditions such that the perceived distance of the character while performing the confrontational behaviour was comparable, in order to eliminate proximity as a differential factor.

We used the following procedure to balance the perceived proximity. We initially chose an observation position for the participant during the VR condition which was approximately $46 \mathrm{~cm}$ from the character, while it was performing the confrontational behaviour. This approximately corresponds to the transition distance from the "personal" to "intimate" proxemic zones ([17]), and is approximately an arms length. To create the perception of a similar separation in the $2 \mathrm{D}$ condition, we placed the virtual camera in Unity3D such that the character's final position was the same distance from the camera as the projection plane, creating a 1:1 scaling at that separation. This was computed using the equation:

$$
d=\frac{h}{2 \times \tan (f \times 0.5)}
$$

Where $d$ is the distance of the virtual camera from the final position of the character, $h$ is the vertical frustum height, set equal to the height of the TV screen used for the study, and $f$ is the field of view of the camera in radians $(\pi \div 3$ in our case). We then marked a viewing position for participants such that their distance from 
the screen was the same as the virtual separation in VR $(46 \mathrm{~cm})$. Finally, we set the height of the virtual camera such that it was the same as the centre of the 2D screen height (approximately $164 \mathrm{~cm}$ ) We measured the height of the character as it appeared on the 2D screen, from floor level, and found it was comparable to the model height in Unity3D $(178 \mathrm{~cm})$. Thus, the perceived distance and height of the character was similar in both conditions.

\subsection{Measures}

In order to address RQ1 we used the State Trait Anxiety Inventory (STAI) self-report measure, Part Y1. This was applied before and after both study conditions, and specifically records state anxiety: we chose it as it best captures the emotional response we anticipated from participants. To address RQ2 we used the ITC Sense of Presence Inventory (ITC-SOPI) self report measure of presence [21], applied after both study conditions. We selected this scale as it is has been designed and used with different media types, and so we consider it most suitable for comparing VR with 2D display experiences.

Finally we conducted a brief post-condition semi-structured interview with all participants, primarily to explore their sense of character believability during the conditions. We asked participants how they felt during the experience, whether they found the character believable, and why (or why not). We also asked for suggestions to make the character more believable. Interviews were voice recorded, and later transcribed.

\subsection{Hypotheses}

With reference to the research questions and measures used, we proposed the following hypotheses. $\mathrm{H} 1$ and $\mathrm{H} 2$ relate to RQ1, and $\mathrm{H} 3$ and H4 relate to RQ2. RQ3 is explored through analyse of the interview data.

- H1: Participants in both conditions will experience an elevated sense of anxiety post-condition, evidenced by pairwise differences in STAI scores. This is motivated by previous results in $2 \mathrm{D}$ [2].

- H2: Participants in the VR condition will experience a larger elevation in anxiety than those in the $2 \mathrm{D}$ condition, motivated by previous result on emotional responses in VR in other contexts (e.g. [11, 16, 19])

- H3: Participants will report higher scores on all subscales (with the exception of negative effects) of the ITC-SOPI in the VR condition, as compared to $2 \mathrm{D}$, based on previous work [11].

- H4: Scores for subscales of the ITC-SOPI in VR will correlate positively with reported elevation in anxiety, suggested by previously results in other contexts (e.g. $[15,38])$.

\subsection{Procedure}

Our study procedure was approved by the ethics committee of the College of Science at the University of Lincoln. We recruited 73 participants by word of mouth, mainly from the campus of the University of Lincoln, all of whom undertook one of the two conditions. Data from five participants was excluded, either because it was incomplete, or due to procedural error. The remaining 68 participants comprised 42 males and 26 females, and ranged in age from 18 to 53 years old $(M=23.78 \mathrm{SD}=6.35)$. Seven reported that they had not used VR before (of which four undertook the 2D condition).

Participants were evenly assigned to the two study conditions to balance for gender, such that each group comprised 21 males and 13 females. All participants were screened for uncorrected vision impairments, and other issues which might impede their ability to use VR equipment safely. Participants then read through an information sheet which outlined the procedure, explained that they would be observing a virtual character performing confrontational behaviour, and their right to withdraw at any time. After reading the information sheet, the researcher confirmed verbally that participants understood the nature of the experience, and that they would like to continue. They then provided written consent. Before undertaking the condition, participants provided demographic data, and completed section $\mathrm{Y} 1$ of the STAI.

Participants in the VR condition experienced the simulation using an HTC Vive Pro system. They were shown how to use the HMD and controllers safely, and they then spent a few minutes in an neutral, empty, VR simulation, where they were familiarised with the equipment setup. Participants in the 2D condition viewed the simulation on a Sony 55" BRAVIA 4K Colour LED Display Television, mounted such that the centre of the TV was approximately $164 \mathrm{~cm}$ from the floor. Participants experienced the simulation audio using bluetooth headphones, in both conditions. The setup for the study conditions is shown in Figure 3

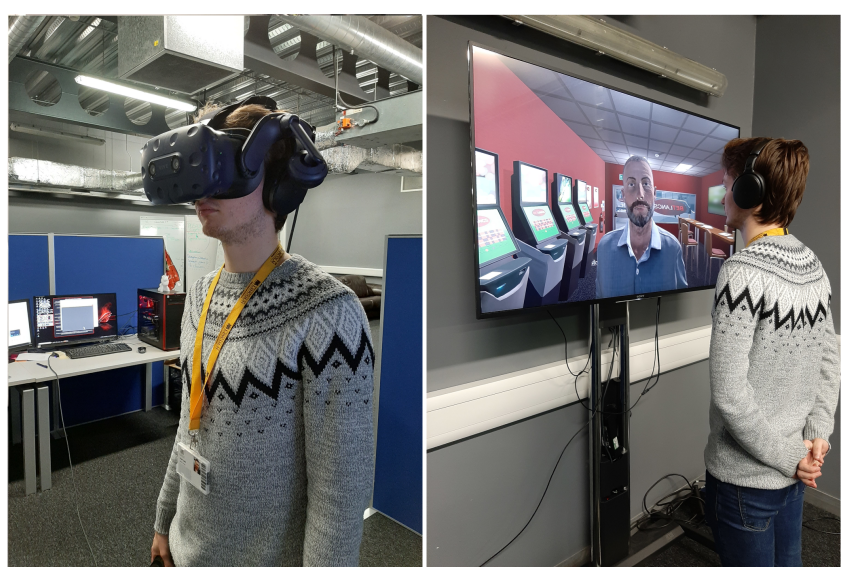

Figure 3: Setup for our study conditions

The scenario presented in the study condition was then explained to participants. They were told that in the simulation they were playing the role of an employee in a betting shop, and were given the task of observing the behaviour of the customer in the shop from a specific standing position (for both conditions, this was indicated on the floor of the laboratory by a cross). Participants were asked if they had any questions, and then entered the condition, which lasted approximately three minutes.

On completing the experience, participants removed the HMD (in the VR condition), and were asked to first complete section Y1 of the STAI again. They then completed the ITC-SOPI questionnaire, and the short semi-structured interview. Participants were finally 
provided with debriefing information, which included contact information for victim support agencies, and given the opportunity to ask any questions they had about the study. The total duration of the procedure was approximately 30-35 minutes for the 2D condition, and 40-45 minutes for the VR condition.

\section{RESULTS}

We report quantitative results obtained from analysis of the STAI and ITC-SOPI data (using IBM SPSS v26), and also qualitative results obtained from analysis of interview data.

\subsection{Results for Self Reported State Anxiety}

To test H1 (participants in both conditions would experience an elevated sense of anxiety) we compared reported anxiety pre and post-condition, for each condition separately, using paired t-tests. Our data met the t-test assumptions, using boxplots to test for outliers, and the Shapiro-Wilk test for normality. In the 2D condition, there was a statistically significant difference between the post-condition scores $(\mathrm{M}=42.71, \mathrm{SD}=11.81)$ and pre-condition scores $(M=32.26, \mathrm{SD}=8.93)$ of $10.44(95 \% \mathrm{CI}, 6.55$ to 14.34$), \mathrm{t}(33)$ $=5.454, \mathrm{p}<0.001$. In the VR condition, there was also a statistically significant difference between the post-condition scores $(M=49.59$, $\mathrm{SD}=11.33)$ and pre-condition scores $(\mathrm{M}=28.74, \mathrm{SD}=6.82)$ of 20.85 (95\% CI, 17.08 to 24.63$), t(33)=11.234, \mathrm{p}<0.001$. These results support hypothesis H1. Distributions of scores, before and after both conditions, are shown in Figure 4.

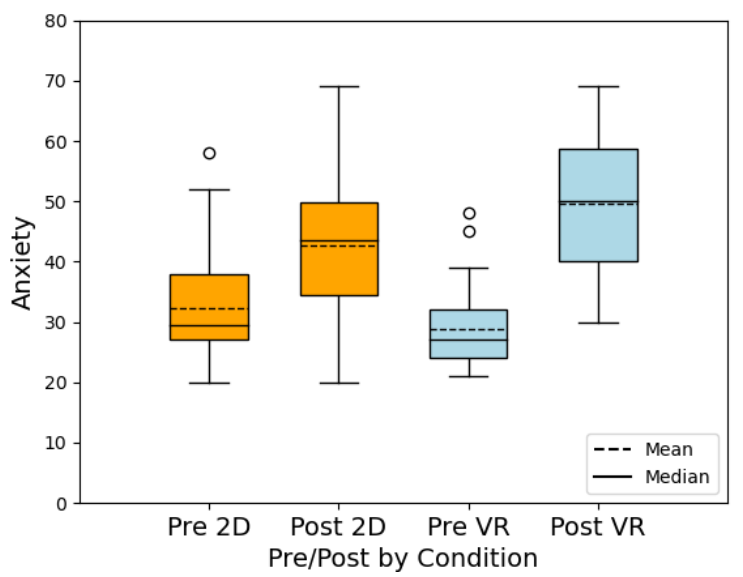

Figure 4: Distributions of STAI scores before and after the 2D and VR conditions.

To test H2 (a higher elevation in anxiety would be recorded after the VR condition), we compared the effects of condition on reported changes in anxiety. As both pre-condition groups were significantly skewed, we opted not to use a two-way mixed ANOVA to examine interaction of the independent variables, and instead used the equivalent method of comparing the mean change in anxiety between the two conditions using an independent means $\mathrm{t}$-test. All assumptions of the t-tests were met. The VR condition $(M=20.85, S D=10.82)$ reported a higher elevation in anxiety than the $2 \mathrm{D}$ condition $(\mathrm{M}=10.44, \mathrm{SD}=11.16)$, representing a statistically significant difference of 10.41 (95\% CI, 5.09 to 15.74$), \mathrm{t}(66)=-3.904$, $\mathrm{p}<0.001$. This result supports hypothesis $\mathrm{H} 2$.

\subsection{Results for Self Reported Presence}

The ITC-SOPI comprises four subscales: sense of physical space, engagement, ecological validity, and negative effects. We note that the sense of physical space subscale broadly corresponds to concepts of spatial presence in the VR literature [21]. For each subscale, we compared data between conditions using independent means t-tests (or equivalent, as detailed).

Reported sense of physical space was higher for the VR condition $(\mathrm{M}=3.76, \mathrm{SD}=0.49)$ than for the $2 \mathrm{D}$ condition $(\mathrm{M}=2.56, \mathrm{SD}=0.68)$. Using Levene's test, sample variances were significantly different $(\mathrm{p}=0.035)$, and so we applied Welch's t-test which confirmed that the difference between the means (95\% CI 0.91 to 1.49$)$ was statistically significant, $\mathrm{t}(60.21)=8.375, \mathrm{p}<0.001$. Engagement was higher for the VR condition $(M=3.68, S D=0.56)$ than for the $2 \mathrm{D}$ condition $(M=3.11$, $\mathrm{SD}=0.61)$. Assumptions of the independent means t-test were met, and the difference of 0.57 (95\% CI 0.29 to 0.85 ) was statistically significant, $\mathrm{t}(66)=4.010, \mathrm{p}<0.001$.

We identified a number of outliers in the data for ecological validity. However, the other assumptions for t-test were met, and we confirmed that the outliers had no significant effect, so retained them. Ecological validity was higher for the VR condition $(\mathrm{M}=4.11$, $\mathrm{SD}=0.49)$ than for $2 \mathrm{D}(\mathrm{M}=3.23, \mathrm{SD}=0.66)$, and the difference $(95 \%$ CI 0.59 to 1.16 ) was statistically significant, $t(66)=6.199, \mathrm{p}<0.001$. The data for negative effects was skewed, so we applied the MannWhitney U test instead, which indicated no statistically significant difference in the distributions for $2 \mathrm{D}$ and $\mathrm{VR}, \mathrm{U}=640, \mathrm{p}=0.447$. Data for the subscales are visualised in Figure 5. Thus, statistically significant higher values were reported for the VR condition across all subscales except negative effects, supporting hypothesis $\mathrm{H} 3$.

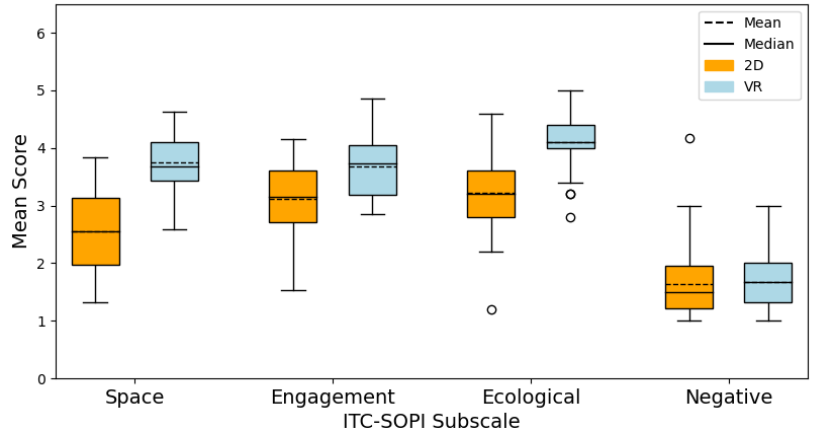

Figure 5: Subscales of the ITC-SOPI

4.2.1 Relationship between Presence and Anxiety. We computed Pearson correlations between reported subscales of the ITC-SOPI and elevation in anxiety, for both VR and 2D conditions. We hypothesised a positive correlation in VR (H4), and computed 1-tailed significance tests at the significance level $\mathrm{p}<0.05$. Results are shown in Table 1 . The only significant correlation found was for sense of physical space, in the VR condition, $\mathrm{r}(32)=0.31, \mathrm{p}=0.037$. This result partially supports hypothesis $\mathrm{H} 4$. 


\begin{tabular}{|c|c|c|}
\hline Subscale & VR & 2D \\
\hline Sense of physical space & $\mathbf{r}=\mathbf{0 . 3 1 1}$ & $\mathrm{r}=0.126$ \\
& $\mathbf{p}=\mathbf{0 . 0 3 7}$ & $\mathrm{p}=0.239$ \\
\hline Engagement & $\mathrm{r}=0.180$ & $\mathrm{r}=0.053$ \\
& $\mathrm{p}=0.154$ & $\mathrm{p}=0.383$ \\
\hline Ecological validity & $\mathrm{r}=0.247$ & $\mathrm{r}=0.140$ \\
& $\mathrm{p}=0.79$ & $\mathrm{p}=0.216$ \\
\hline Negative effects & $\mathrm{r}=-0.022$ & $\mathrm{r}=0.271$ \\
& $\mathrm{p}=0.451$ & $\mathrm{p}=0.060$ \\
\hline
\end{tabular}

Table 1: Pearson Correlations between Elevation in Anxiety and Subscales of the ITC-SOPI

\subsection{Qualitative Results}

Participant interviews were brief, typically lasting 2-4 minutes, and transcriptions were imported into NVivo v12. We analysed the data using an inductive thematic analysis, following the approach outlined by Braun and Clarke [7]. Initial coding indicated a high degree of similarity between nodes from each condition, so we proceeded to analyse the data from both conditions together (we report differences where pertinent). We identified 130 nodes across the whole data set, from which we developed five primary themes which capture participants' views on their experience, and their perceptions of character believability.

Theme 1: Relating and Responding to the Character's Behaviour. Participants overwhelmingly referred to the character's behaviour positively when discussing aspects of believability. This was a dominant theme, and participants picked out and described features of the build up of frustration positively, suggesting that they found this relatable. For example "His build up reaction was exactly what I would expect, and what I have experienced... it started off being internally frustrated, and started signs of exhibiting small amounts of frustration". Participants also described anticipating the outcome of the behaviour as it developed, for example "I could tell that they were like building up emotion, and I could tell that they were going to be set off, so I was kind of like preparing myself for that". Several mentioned that the glances the character made towards them while playing enhanced their sense of believability.

A number participants drew directly on their real-life experiences when discussing the behaviour. One commented "He reminds me of a lot of when I used to work in retail, that you would actually have customers like that, that we would sometimes fly off handle like that, and actually kicked in how I would actually respond." which suggests that their real-life experiences reinforced their sense of believability. A small number of participants also reflected on their own physical responses, such as stepping away from the character, or experiencing a sensation of threat. For example, one participant remarked "I felt a bit scared like, like it could touch me in some way as well", and another "my body's response, even though my brain knew it was fake, was to lean back a little bit to try and get away".

Theme 2: Visual appearance undermining believability. When talking about factors which reduced believability, participants predominantly mentioned visual aspects of the experience, including pixel resolution and textures. For example, one participant said "it wasn't quite photo realistic, you know, it was quite obviously, like, a digital avatar. But I don't know how much more realistic it could be.", which suggests that some participants may have equated believability with realism. There were also comments related to minor errors in animation or perceived unnaturalness of particular movements, for example "I guess like some of the movements seemed a bit like, more like, jolty than like in real-life: like it didn't seem as fluid, slightly". However, there were also some positive comments about the character's animations; for example, identifying eye movements as being believable. Some participants referred to visual appearance when suggesting ways to improving overall believability.

Theme 3: Variety of Experience. The majority of participants described their experience using words like "uncomfortable", "anxious", "tense" or "stressful", which suggests similar responses of varying intensity, and also sometimes linked these to specific aspects of the experience, such as the proximity of the character. However, a number of participants described their experience in other ways, such as feeling disconnected or removed from the scene. For example "Neutral, really... didn't really feel anything because it was quite separated from the actual, real world". The majority of these participants experienced the $2 \mathrm{D}$ condition. However, a number of VR participants also reported such feelings. A small number of participants also reported other types of responses which were less predictable, such as humour, curiosity, or feelings of empathy.

Theme 4: Interactivity Limiting Believability. Some participants commented on the interactivity of their experience, generally indicating that more interactivity could improve believability, and a number of specific suggestions were made. For example, "there weren't any other people, so there wasn't anyone else to interact with that would maybe sort of see his behaviour and either do something before it happened, or that sort of thing". A few of participants also mentioned that a lack of physical threat reduced believability. One remarked "he was coming out to me but because I know he's not real and he cannot touch me so it's not really, like real.". However, some comments also suggested that aspects of the experience were quite interactive (such as the character looking at them), and added to believability.

Theme 5: Audio Enhancing Experience. Several participants mentioned aspects of the scene audio, predominantly in a positive way, when discussing believability. In particular, several specifically mentioned the character's voice, and the "voice acting". A number of suggestions were also made as to how audio could enhance believabilty, such as more talking during the early parts of the experience; for example, "I suppose from a certain distance you're not gonna hear like mutterings... but maybe something like that just to, just to, I don't know maybe build your attention"

\section{DISCUSSION}

The use of VR for training applications (or indeed other experiences) which involve simulating confrontational behaviour has received relatively little attention. Our work therefore has some implications both for further research, and for the creators of such experiences, which we present and discuss here. 


\subsection{Creating Believable Characters}

Existing discussions around the believability of VR characters have mainly centred around perceived intelligence [32], graphical representation, and the "uncanny valley" effect [51]. However, little work has considered believability in the context of confrontational behaviour. Previous work by Slater et al. [44], in their VR study of pro-social behaviour, raised issues around credibility of the setting, dialogue, and interactivity. In contrast, our participants reported that their perceptions of the character's believability were enhanced by features of its behaviour, and several related to it through the lens of previous real-life experiences. Together this suggests that convincing character behaviour is an important factor in users' experience of simulated confrontations, and we have provided insights into how such behaviours may be produced using performance-based animation, and domain-specific knowledge of human behaviour.

In our qualitative analysis, themes relating to behaviour and interactivity resonate with Slater's concept of plausibility illusion [42], suggesting that participants' own perceptions of character believability overlap with established concepts of presence in VR. While this is not necessarily surprising, these themes were evident across both conditions. We also identified themes of graphical and audio quality, providing a set of key reference points for the creators of similar experiences.

We also identified interactivity as a theme, and previous work has highlighted that perceived intelligence and responsiveness is associated with believability [32]. Since our behaviour was almost entirely pre-animated (with the exception of eye movement), this seems to present a choice between production processes (mocap or real-time animation), both of which may influence believability positively. However, we argue that these approaches are complementary: optimal design may involve considered trade-offs, or a blend of automated and pre-animated behaviours, to create the best user experience. Thus, we posit that the integration of domain knowledge and performative elements, with real-time behaviours, presents a desirable goal for future research. We also highlight that in our study, which had limited interactivity, participants were passive "receivers" of confrontational behaviour. In a fully interactive experience, where users can respond and take control of the situation, we might expect that their perceptions of their experience might be more complex. Thus, we consider that the responses reported by our participants emphasise one aspect of such experiences, and also invite further investigation in future work.

\subsection{Platform Choices}

Participants reported a higher elevation in anxiety in VR, providing justification for using VR where evoking a realistic emotional response is an important outcome. We also show that a less intense elevation is achievable using a 2D display (mirroring [2]), which presents possible trade-offs for designers; for example, novice users might experience training scenarios initially in $2 \mathrm{D}$, and later in VR, to create a gradient of experience. However, we have not compared VR with an equivalent real-life experience, so whether the response in VR is comparable remains an open question.

Participants in VR also reported a higher sense of presence, which is in line with previous work comparing levels of immersion [11].
We note that although negative effects was reported as low in both conditions, our study conditions are short, and we therefore do not draw any conclusions about longer exposure. Of more interest is the correlation between sense of physical space and anxiety, reported in the VR condition, which parallels some previous work in VR exposure therapy [15]. The relationship between presence and emotional response in VR is an active area of research, and no sense of causality is inferred; however, since this correlation occurs on sense of physical space, we conjecture that anxiety might be mediated by a sense of proximity with the character, since it is known that close proximity can induce discomfort in VR [14, 23, 50].

\subsection{Ethical Considerations}

Ethical issues around creating potentially stressful situations in VR have been discussed in the literature (e.g. [37]). Our study shows that confrontational virtual characters in VR can lead to a significant emotional response in users (more so than on a 2D screen). None of our participants reported or showed signs of distress; however, this may be because they were informed about the nature of the experience, engagement was brief, and the character did not directly threaten them. We therefore emphasise two points. Firstly, researchers and practitioners used to working with 2D displays may consider that, when moving to VR, appropriate mitigations may be necessary to avoid accidental distress. Secondly, participants may not have used VR before, and so not understand the differences between experiences in VR, and those on a 2D screen. Whilst they will understand that VR experiences are not real, they may not appreciate that they can evoke significant emotional reactions.

Likewise, we believe that our findings have implications for longer-term immersion, and where users encounter unexpected instances of confrontation. Here, guidance may be required to support users while they engage with the experience, when they exit VR, and during debriefing. These are issues which institutional ethics committees, as well as researchers, may wish to consider.

\subsection{Limitations and Future Work}

Our work has a number of limitations which we outline as follows, along with suggestions for future extensions. Firstly, as we noted in the previous section, we have not compared VR directly with an equivalent real-life performance, and we therefore propose that this would be a revealing future study. Regarding believability, and with reference to Section 4.3, we highlight that participants sometimes appeared to conflate believability and visual realism, when describing their experience. We note that these are distinct concepts, and that visual realism may also affect presence in VR [43]; a more detailed exploration and differentiation of these concepts is warranted, which could also be addressed in future work. In addition, our sequence was relatively short, lasting only a few minutes, and, while representative of the contextualised behaviour that we wished to portray, results for longer exposures, that involve interactions in confrontational situations, may reveal further issues affecting perceived believability.

We also note that although we have presented our work within the context of training applications, our participants were not trainees. This gives us a limited perspective on applicability to fully developed training applications, as our participants can only 
be considered as representative of naive or new users. Thus, as future work related specifically to application in training, we propose developing a set of longer experiences which could be used in more detailed studies with users at different levels of experience, and possibly in different training contexts.

Our motion capture process seemed effective, but did pose some technical challenges. Some of the performed movements were erratic and fast moving, and in some cases the captured motions were noisy, contained processing errors, or on some occasions, mocap markers became detached. Although we were able to correct most errors during post-processing, some remained and were subsequently noticed by a few participants when discussing believability. We therefore consider that the use of higher fidelity motion capture techniques in future studies would be beneficial, and help to reveal a deeper understanding of users' perceptions of believability. Finally, having noted that interactivity and perceived intelligence is also a factor in character believability, we propose that, as a longterm goal, future work on the development of intelligent characters might seek to integrate elements of our approach with real-time AI techniques, with the objective of creating characters which are responsive, performative, and capture domain knowledge.

\section{CONCLUSION}

We have conducted a user study to compare self-reported elevation in anxiety between two groups of participants who experienced a virtual character simulating confrontational behaviour: one group experienced the behaviour in VR, and the other on a 2D display. Our results showed a statistically significant elevation for the $2 \mathrm{D}$ condition, supporting previous results by Blankendaal et al. [2]. However, a higher level of elevation was seen for the VR condition, suggesting that VR may provide a more effective platform for training (or other) applications which seek to evoke a realistic response to simulations of confrontational situations. We additionally saw a higher level of reported sense of presence in the VR condition, and also a significant correlation between the sense of physical space subscale of the ITC-SOPI, and reported elevation in anxiety, also in VR.

Some previous work has shown that the credibility of such simulations may undermine users' experiences of them. We therefore also collected qualitative data, to explore factors affecting participants' sense of character believability. This revealed that visual factors such as graphical quality and animation errors were the most commonly reported negative factors, whereas the character's behaviour was mainly considered to contribute positively. Based on these results we have argued for the use of domain-specific knowledge of human behaviour, and motion captured performance, to be used in the creation of such experiences, and also for the use of VR platforms in their deployment, particularly in training contexts.

\section{REFERENCES}

[1] Ivan Alsina-Jurnet, José Gutiérrez-Maldonado, and María-Virgina Rangel-Gómez. 2011. The role of presence in the level of anxiety experienced in clinical virtual environments. Computers in Human Behavior 27, 1 (2011), 504 - 512. https: //doi.org/10.1016/j.chb.2010.09.018 Current Research Topics in Cognitive Load Theory.

[2] Romy Blankendaal, Tibor Bosse, Charlotte Gerritsen, Tessa de Jong, and Jeroen de Man. 2015. Are Aggressive Agents As Scary As Aggressive Humans? In Proceedings of the 2015 International Conference on Autonomous Agents and Multiagent Systems (Istanbul, Turkey) (AAMAS '15). International Foundation for
Autonomous Agents and Multiagent Systems, Richland, SC, 553-561.

[3] Tibor Bosse, Charlotte Gerritsen, and Jeroen de Man. 2016. An Intelligent System for Aggression De-escalation Training. In Proceedings of the Twenty-second European Conference on Artificial Intelligence (The Hague, The Netherlands) (ECAI'16). IOS Press, Amsterdam, The Netherlands, The Netherlands, 1805-1811. https://doi.org/10.3233/978-1-61499-672-9-1805

[4] Tibor Bosse, Tilo Hartmann, Romy A.M. Blankendaal, Nienke Dokter, Marco Otte, and Linford Goedschalk. 2018. Virtually Bad: A Study on Virtual Agents That Physically Threaten Human Beings. In Proceedings of the 17th International Conference on Autonomous Agents and MultiAgent Systems (Stockholm, Sweden) (AAMAS '18). International Foundation for Autonomous Agents and Multiagent Systems, Richland, SC, 1258-1266.

[5] Stéphane Bouchard, Julie St-Jacques, Geneviève Robillard, and Patrice Renaud. 2008. Anxiety Increases the Feeling of Presence in Virtual Reality. Presence: Teleoperators and Virtual Environments 17, 4 (2008), 376-391. https://doi.org/10. 1162/pres.17.4.376

[6] Cheryl Campanella Bracken. 2005. Presence and Image Quality: The Case of High-Definition Television. Media Psychology 7, 2 (2005), 191-205. https://doi. org/10.1207/S1532785XMEP0702 4

[7] Virginia Braun and Victoria Clarke. 2006. Using thematic analysis in psychology. Qualitative Research in Psychology 3, 2 (2006), 77-101. https://doi.org/10.1191/ 1478088706qp063oa

[8] A. Bönsch, S. Radke, H. Overath, L. M. Asché, J. Wendt, T. Vierjahn, U. Habel, and T. W. Kuhlen. 2018. Social VR: How Personal Space is Affected by Virtual Agents' Emotions. In 2018 IEEE Conference on Virtual Reality and 3D User Interfaces (VR). 199-206. https://doi.org/10.1109/VR.2018.8446480

[9] Emily Carl, Aliza T. Stein, Andrew Levihn-Coon, Jamie R. Pogue, Barbara Rothbaum, Paul Emmelkamp, Gordon J.G. Asmundson, Per Carlbring, and Mark B. Powers. 2019. Virtual reality exposure therapy for anxiety and related disorders: A meta-analysis of randomized controlled trials. Fournal of Anxiety Disorders 61 (2019), 27 - 36. https://doi.org/10.1016/j.janxdis.2018.08.003 Virtual reality applications for the anxiety disorders.

[10] Gordon Carlson and Nicholas Caporusso. 2019. A Physically Immersive Platform for Training Emergency Responders and Law Enforcement Officers. In Advances in Human Factors in Training, Education, and Learning Sciences. Springer International Publishing, 108-116.

[11] James J. Cummings and Jeremy N. Bailenson. 2016. How Immersive Is Enough? A Meta-Analysis of the Effect of Immersive Technology on User Presence. Media Psychology 19, 2 (2016), 272-309. https://doi.org/10.1080/15213269.2015.1015740

[12] Elise Dan-Glauser and Klaus Scherer. 2011. The Geneva affective picture database (GAPED): A new 730-picture database focusing on valence and normative significance. Behavior research methods 43 (03 2011), 468-77. https: //doi.org/10.3758/s13428-011-0064-1

[13] Aline W. de Borst and Beatrice de Gelder. 2015. Is it the real deal? Perception of virtual characters versus humans: an affective cognitive neuroscience perspective. Frontiers in Psychology 6 (2015), 576. https://doi.org/10.3389/fpsyg.2015.00576

[14] Patrick Dickinson, Kathrin Gerling, Kieran Hicks, John Murray, John Shearer, and Jacob Greenwood. 2019. Virtual reality crowd simulation: effects of agent density on user experience and behaviour. Virtual Reality 23, 1 (01 Mar 2019), 19-32. https://doi.org/10.1007/s10055-018-0365-0

[15] Julia Diemer, Georg W. Alpers, Henrik M. Peperkorn, Youssef Shiban, and Andreas Mühlberger. 2015. The impact of perception and presence on emotional reactions: a review of research in virtual reality. Frontiers in Psychology 6 (2015), 26. https: //doi.org/10.3389/fpsyg.2015.00026

[16] Sergio Estupiñán, Francisco Rebelo, Paulo Noriega, Carlos Ferreira, and Emília Duarte. 2014. Can Virtual Reality Increase Emotional Responses (Arousal and Valence)? A Pilot Study. In Design, User Experience, and Usability. User Experience Design for Diverse Interaction Platforms and Environments, Aaron Marcus (Ed.). Springer International Publishing, 541-549.

[17] Edward T. Hall. 1963. A System for the Notation of Proxemic Behavior1. American Anthropologist 65, 5 (1963), 1003-1026. https://doi.org/10.1525/aa.1963.65.5. 02a00020

[18] Matthias Hoppe, Beat Rossmy, Daniel Peter Neumann, Stephan Streuber, Albrecht Schmidt, and Tonja-Katrin Machulla. 2020. A Human Touch: Social Touch Increases the Perceived Human-Likeness of Agents in Virtual Reality (CHI '20). Association for Computing Machinery, New York, NY, USA, 1-11. https://doi.org/10.1145/3313831.3376719

[19] Kwanguk Kim, M. Zachary Rosenthal, David J. Zielinski, and Rachael Brady. 2014. Effects of virtual environment platforms on emotional responses. Computer Methods and Programs in Biomedicine 113, 3 (2014), 882 - 893. https://doi.org/10. 1016/j.cmpb.2013.12.024

[20] Marios Kyriakou, Xueni Pan, and Yiorgos Chrysanthou. 2017. Interaction with virtual crowd in Immersive and semi-Immersive Virtual Reality systems. Computer Animation and Virtual Worlds 28, 5 (2017), e1729. https://doi.org/10.1002/cav.1729

[21] J. Lessiter, J. Freeman, E. Keogh, and J. Davidoff. 2001. A Cross-Media Presence Questionnaire: The ITC-Sense of Presence Inventory. Presence 10, 3 (June 2001), 282-297. 
[22] Yun Ling, Willem-Paul Brinkman, Harold T. Nefs, Chao Ou, and Ingrid Heynderickx. 2012. Effects of Stereoscopic Viewing on Presence, Anxiety, and Cybersickness in a Virtual Reality Environment for Public Speaking. Presence: Teleoperators and Virtual Environments 21, 3 (2012), 254-267.

[23] Joan Llobera, Bernhard Spanlang, Giulio Ruffini, and Mel Slater. 2010. Proxemics with Multiple Dynamic Characters in an Immersive Virtual Environment. ACM Tranactions on Applied Perception 8, 1 (Nov. 2010), 3:1-3:12. https://doi.org/10. 1145/1857893.1857896

[24] Sarah Lopez, Yi Yang, Kevin Beltran, Soo Jung Kim, Jennifer Cruz Hernandez, Chelsy Simran, Bingkun Yang, and Beste F. Yuksel. 2019. Investigating Implicit Gender Bias and Embodiment of White Males in Virtual Reality with Full Body Visuomotor Synchrony. In Proceedings of the 2019 CHI Conference on Human Factors in Computing Systems (Glasgow, Scotland Uk) (CHI '19). Association for Computing Machinery, New York, NY, USA, 1-12. https://doi.org/10.1145/ 3290605.3300787

[25] R. McGloin and M. Krcmar. 2011. The Impact of Controller Naturalness on Spatial Presence, Gamer Enjoyment, and Perceived Realism in a Tennis Simulation Video Game. Presence 20, 4 (Aug 2011), 309-324. https://doi.org/10.1162/PRES a 00053

[26] R. P. McMahan, D. A. Bowman, D. J. Zielinski, and R. B. Brady. 2012. Evaluating Display Fidelity and Interaction Fidelity in a Virtual Reality Game. IEEE Transactions on Visualization and Computer Graphics 18, 4 (April 2012), 626-633. https://doi.org/10.1109/TVCG.2012.43

[27] Johannes Moskaliuk, Johanna Bertram, and Ulrike Cress. 2013. Training in virtual environments: putting theory into practice. Ergonomics 56, 2 (2013), 195-204. https://doi.org/10.1080/00140139.2012.745623

[28] Sahil Narang, Andrew Best, Tanmay Randhavane, Ari Shapiro, and Dinesh Manocha. 2016. PedVR: Simulating Gaze-based Interactions Between a Real User and Virtual Crowds. In Proceedings of the 22Nd ACM Conference on Virtual Reality Software and Technology (Munich, Germany) (VRST '16). ACM, New York, NY, USA, 91-100. https://doi.org/10.1145/2993369.2993378

[29] A. Olivier, J. Bruneau, R. Kulpa, and J. Pettré. 2018. Walking with Virtual People: Evaluation of Locomotion Interfaces in Dynamic Environments. IEEE Transactions on Visualization and Computer Graphics 24, 7 (July 2018), 2251-2263. https://doi.org/10.1109/TVCG.2017.2714665

[30] Select Committee on the Social and Economic Impact of the Gambling Industry 2020. Gambling Harm - Time for Action (HL 2019-2021, 79).

[31] Xueni Pan, Marco Gillies, and Mel Slater. 2015. Virtual Character Personality Influences Participant Attitudes and Behavior - An Interview with a Virtual Human Character about Her Social Anxiety. Frontiers in Robotics and AI 2 (2015) https://doi.org/10.3389/frobt.2015.00001

[32] Xueni Pan and Antonia F. de C. Hamilton. 2018. Why and how to use virtual reality to study human social interaction: The challenges of exploring a new research landscape. British fournal of Psychology 109, 3 (2018), 395-417. https: //doi.org/10.1111/bjop.12290

[33] Xueni Pan, Mel Slater, Alejandro Beacco, Xavi Navarro, Anna I Bellido Rivas, David Swapp, Joanna Hale, Paul Alexander George Forbes, Catrina Denvir, Antonia F. de C. Hamilton, and Sylvie Delacroix. 2016. The Responses of Medical General Practitioners to Unreasonable Patient Demand for Antibiotics-A Study of Medical Ethics Using Immersive Virtual Reality. PloS ONE 11, 2 (February 2016), e0146837.

[34] Adrian Parke and Mark Griffiths. 2004. Aggressive Behaviour in Slot Machine Gamblers: A Preliminary Observational Study. Psychological Reports 95, 1 (2004), 109-114. https://doi.org/10.2466/pr0.95.1.109-114

[35] Adrian Parke and Mark Griffiths. 2005. Aggressive behaviour in adult slotmachine gamblers: A qualitative observational study. eCOMMUNITY: International fournal of Mental Health Addiction 2, 2 (2005), 50-58.

[36] Adrian Parke and Mark Griffiths. 2005. Aggressive behaviour in adult slot machine gamblers: an interpretative phenomenological analysis. Fournal of Community \& Applied Social Psychology 15, 4 (2005), 255-272. https://doi.org/10.1002/casp.824

[37] Henrik M. Peperkorn, Georg W. Alpers, and Andreas Mühlberger. 2014. Triggers of Fear: Perceptual Cues Versus Conceptual Information in Spider Phobia. Journal of Clinical Psychology 70, 7 (2014), 704-714. https://doi.org/10.1002/jclp.22057

[38] Giuseppe Riva, Fabrizia Mantovani, Claret Samantha Capideville, Alessandra Preziosa, Francesca Morganti, Daniela Villani, Andrea Gaggioli, Cristina Botella, and Mariano Alcañiz. 2007. Affective Interactions Using Virtual Reality: The Link between Presence and Emotions. CyberPsychology \& Behavior 10, 1 (2007), 45-56. https://doi.org/10.1089/cpb.2006.9993

[39] Aitor Rovira, David Swapp, Bernhard Spanlang, and Mel Slater. 2009. The Use of Virtual Reality in the Study of People's Responses to Violent Incidents. Frontiers in behavioral neuroscience 3 (12 2009), 59. https://doi.org/10.3389/neuro.08.059.2009

[40] Daniel M. Shafer, Corey P. Carbonara, and Lucy Popova. 2011. Spatial Presence and Perceived Reality as Predictors of Motion-Based Video Game Enjoyment. Presence: Teleoperators and Virtual Environments 20, 6 (2011), 591-619. https: //doi.org/10.1162/PRES_a_00084

[41] Richard Skarbez, Frederick P. Brooks, Jr., and Mary C. Whitton. 2017. A Survey of Presence and Related Concepts. ACM Comput. Surv. 50, 6, Article 96 (Nov. 2017), 39 pages. https://doi.org/10.1145/3134301
[42] Mel Slater. 2009. Place illusion and plausibility can lead to realistic behaviour in immersive virtual environments. Philosophical Transactions of the Royal Society B: Biological Sciences 364, 1535 (2009), 3549-3557. https://doi.org/10.1098/rstb. 2009.0138

[43] M. Slater, P. Khanna, J. Mortensen, and I. Yu. 2009. Visual Realism Enhances Realistic Response in an Immersive Virtual Environment. IEEE Computer Graphics and Applications 29, 3 (2009), 76-84. https://doi.org/10.1109/MCG.2009.55

[44] Mel Slater, Aitor Rovira, Richard Southern, David Swapp, Jian J. Zhang, Claire Campbell, and Mark Levine. 2013. Bystander Responses to a Violent Incident in an Immersive Virtual Environment. PLOS ONE 8, 1 (01 2013), 1-13.

[45] Mel Slater, Bernhard Spanlang, Maria Sanchez-Vives, and Olaf Blanke. 2010. First Person Experience of Body Transfer in Virtual Reality. PLoS One 5, 5 (05 2010).

[46] Martin Usoh, Kevin Arthur, Mary C. Whitton, Rui Bastos, Anthony Steed, Mel Slater, and Frederick P. Brooks, Jr. 1999. Walking > Walking-in-place > Flying, in Virtual Environments. In Proceedings of the 26th Annual Conference on Computer Graphics and Interactive Techniques (SIGGRAPH '99). ACM Press/Addison-Wesley Publishing Co., New York, NY, USA, 359-364. https://doi.org/10.1145/311535. 311589

[47] Lucia R. Valmaggia, Leila Latif, Matthew J. Kempton, and Maria Rus-Calafell. 2016. Virtual reality in the psychological treatment for mental health problems: An systematic review of recent evidence. Psychiatry Research 236 (2016), 189 195. https://doi.org/10.1016/j.psychres.2016.01.015

[48] Marnix S. van Gisbergen, Ilay Sensagir, and Joey Relouw. 2020. How Real Do You See Yourself in VR? The Effect of User-Avatar Resemblance on Virtual Reality Experiences and Behaviour. Springer International Publishing, Cham, 401-409. https://doi.org/10.1007/978-3-030-37869-1 32

[49] H. Wardle, A. Moody, S. Spence, J. Orford, R. Volberg, D. Jotangia, M. Griffiths, Hussey D, and F. Dobbie. 2011. British Gambling Prevalence Survey 2010.

[50] Laurie M. Wilcox, Robert S. Allison, Samuel Elfassy, and Cynthia Grelik. 2006. Personal Space in Virtual Reality. ACM Transactions on Applied Perception 3, 4 (Oct. 2006), 412-428. https://doi.org/10.1145/1190036.1190041

[51] K. Zibrek, E. Kokkinara, and R. Mcdonnell. 2018. The Effect of Realistic Appearance of Virtual Characters in Immersive Environments - Does the Character's Personality Play a Role? IEEE Transactions on Visualization and Computer Graphics 24, 4 (2018), 1681-1690. 